\title{
Enhanced Porosity without Compromising Structural Integrity: The Nemesis of Electrospun Scaffolding
}

\section{Gary L. Bowlin}

Department of Biomedical Engineering, Virginia Commonwealth University, Richmond, Virginia

Over the last decade, electrospinning to create non-woven fabrics composed of nano- and micrometer diameters fibers has gone from an unknown process to commonplace in the tissue engineering community. Unfortunately, the majority of the scaffolds fabricated have an extremely limited capacity to promote three-dimensional tissue regeneration. This is because the fine pore structure created in the scaffolding limits cellular infiltration, thus acting more as a pseudo two-dimensional surface for enhanced cell adhesion. Hence the challenge, as the use of electrospinning for fabricating tissue engineering scaffolding moves toward functional, three-dimensional tissue engineered constructs, will be to enhance the overall porosity without compromising overall structural integrity. This is a critical challenge yet to be overcome. If this processing deficiency cannot be corrected, it is highly probable that the process of electrospinning will be considered a failure in developing tissue engineering scaffolds.

A goal of any tissue engineering approach is to develop scaffolding capable of functional regeneration. To duplicate all the essential intercellular reactions and promote native intracellular responses, the tissue engineers' goal is to mimic the native extracellular matrix (ECM). ECM analogues, or scaffolds, should conform to a specific set of requirements [1]. The native structural ECM fibers $(50-300 \mathrm{~nm}$ in diameter) are one to two orders of magnitude smaller than the cell itself, allowing cells to interact with multiple fibers and define cellular 3-D orientation. Thus, engineers have tried to replicate the ECM as a scaffold for tissue development. Without significant porosity, it has been difficult to establish an even 3-D distribution of cells regardless of scaffold fabrication method or cell seeding technique. Electrospinning represents a method to meet the general scaffold requirements, as well as the potential size issues in mimicking the ECM, and has been described extensively in terms of the process [2-4] and its potential applications in tissue engineering $[5,6]$. What has been learned by the electrospinning community to this point is that cells have an extraordinarily difficult time migrating across fibers (penetrating scaffolds) due to the random deposition and packing of fibers (severely restricted by fine pore structure). Those same cells will rapidly migrate along the fibers. Thus, the take home message, we just need to get the cells into the electrospun scaffolds and from there they will do the work, migrating to create an even cellular density fairly rapidly and from there regenerate threedimensional tissue constructs.

In order to get the cells into the scaffolds rapidly, several scaffold fabrication processing variations have been attempted with limited success. One technique attempted to enhance cell seeding and infiltration was the fabrication of electrospun scaffolds composed of both synthetic and natural (native integrin binding sites) polymers [7-10]. While they enhanced cell adhesion, these structures have had limited success in improving cellular infiltration. The use of porogens in electrospun scaffolds has also been utilized with the first study by Zhang et al. in which they electrospun a blended solution of polycaprolactone (PCL) and gelatin without cross-linking which meant a large percentage of the gelatin was dissolved when immersed in an aqueous media [11]. This was followed by Baker et al. in which poly(ethylene oxide) (PEO) fibers were electrospun and intermingled with simultaneously electrospun
PCL fibers with the water-soluble PEO "sacrificial fibers" removed by post-processing [12]. The results showed that the greater the PEO in the scaffold, the greater the cellular infiltration (majority of cells remaining in the upper $25 \%$ of the scaffold) but at the expenses of a significant reduction in the scaffolds' modulus and maximum stress. Nam et al. introduced salt crystals amongst the fibers that were removed by postprocessing to create void areas that enhanced cell infiltration [13]. The major concerns are the uneven distribution of the crystals and loss of scaffold integrity (macroscopic scaffold layer delamination). The use of cryogenic electrospinning is another processing variation that has been attempted. This technique utilizes a mandrel maintained at very low temperatures in a controlled humidity environment to allow for the deposition of ice crystals amongst the fiber deposition which can then be removed by lyophilization, leading to a significant enhancement in the scaffold porosity $[14,15]$. Unfortunately, the tensile strength of the enhanced porosity scaffold is 40 times weaker than scaffolding created on a non-cryogenic mandrel [14]. The most recently published processing modification is the use of a spherical-dish for fiber collection to create an electrospun scaffolding which is "cotton ball-like" [16]. This configuration obviously enhanced cellular three-dimensional infiltration versus traditional electrospinning mandrels but the lack of structural integrity will again severely limit the potential applications of such scaffolding. The overriding theme with the previous attempts at creating enhanced porosity is the significant loss of overall structural integrity. This will be a major limitation in terms of their use in loadbearing tissue engineering applications (in vitro and especially in vivo or in situ) which constitutes a majority of the products targeted.

To overcome the cell infiltration limitations, we have recently introduced a novel electrospinning mandrel to create a more open, porous structure through air-impendence electrospinning. The process modification in this case employs a hollow mandrel with defined pores to allow pressurized air to be expelled through the pores to create air jets that disrupt fiber deposition and prevent compaction of the fibers. As a feasibility study, PCL was electrospun (no airflow or air supplied at $100 \mathrm{kPa}$ ) on the perforated mandrel or a traditional solid mandrel with identical amounts of polymer spun (all other processing parameters constant). Visual inspection shows that the air disturbance method scaffolds have an obvious increase in overall wall thickness versus zero air flow or solid mandrel, demonstrating an increased porosity. The solid mandrel scaffolds have even, uniform surfaces of densely packed fibers. The fibrous nature (densely packed

Corresponding author: Gary L. Bowlin, Department of Biomedical Engineering Virginia Commonwealth University, Richmond, Virginia, Email: glbowlin@vcu.edu

Received April 29, 2011; Accepted April 29, 2011; Published May 06, 2011

Citation: Bowlin GL (2010) Enhanced Porosity without Compromising Structural Integrity: The Nemesis of Electrospun Scaffolding. J Tissue Sci Eng 2:103e. doi:10.4172/2157-7552.1000103e

Copyright: @ 2010 Bowlin GL. This is an open-access article distributed under the terms of the Creative Commons Attribution License, which permits unrestricted use, distribution, and reproduction in any medium, provided the original author and source are credited. 

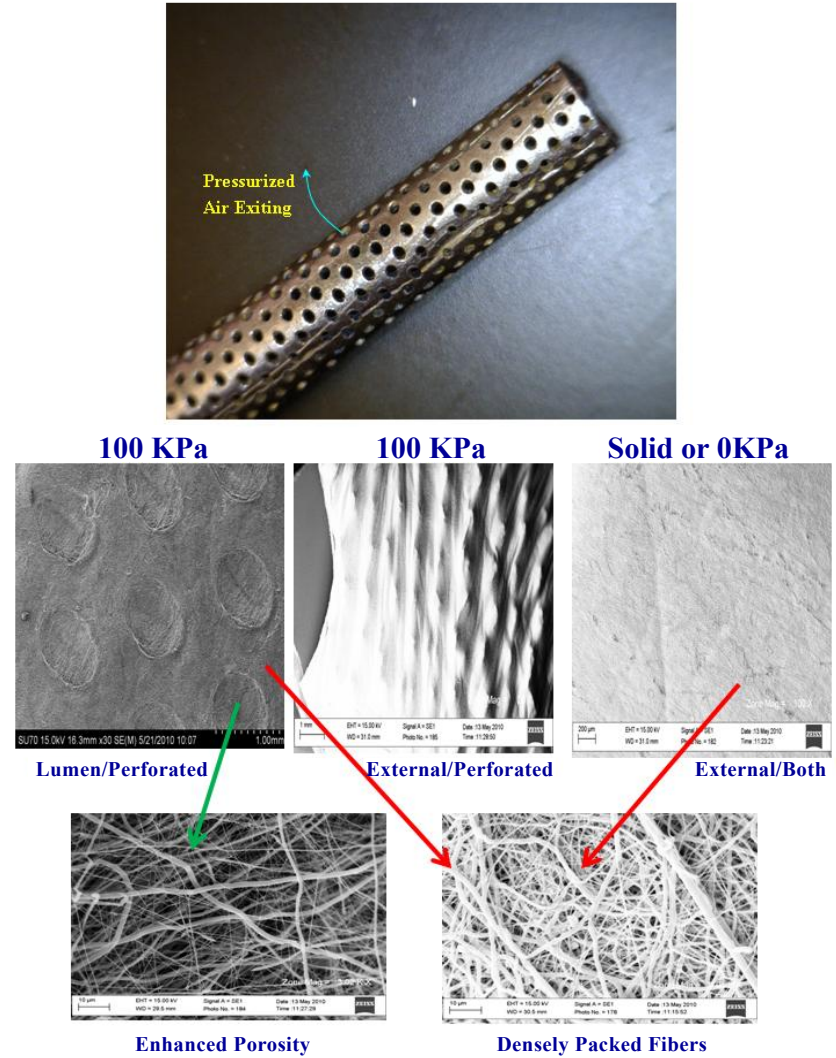

Figure 1:

fibers) of the scaffolds from the perforated mandrel (no air flow, 0 $\mathrm{kPa}$ ) are very similar to the solid mandrel with the exception of the internal surface where the fiber density is less in the areas directly adjacent the open pore sections of the mandrel (electric field effects). For the airflow samples, less dense fiber packing is seen on the internal surface above the pores (between the pores resembles the solid mandrel fiber deposition) and the external surface above the perforated areas with some raised regions; unlike the zero airflow samples resembling the solid mandrel. This indicates an extreme airflow, further process optimization will eliminate this phenomena. Water permeability testing of the scaffolds showed that water permeability at $120 \mathrm{mmHg}$ for the $100 \mathrm{kPa}$ air impedance electrospun scaffolds was twice that of the solid mandrel while the burst strength remained constant for all three scaffolds. This unique structure for the first time, to the best of our knowledge, allows for an increased porosity without compromising the overall mechanical integrity (burst strength) by the combination of dense fiber areas formed between pores imparting strength (loss of this support structure will significantly compromise strength) as well as adding stability to the enhanced porosity (preventing collapse). Thus, the preliminary results have demonstrated that air-flow impedance electrospinning is effective at creating a more porous structure without compromising mechanical integrity. There will be a point where this enhanced porosity will start to degrade mechanical integrity. Thus, the system must be optimized (pore size, pore spacing, air flow rate, etc.) to maximize cellular infiltration and distribution with minimal to no impact on structural integrity. Various static cell seeding studies comparing the air-impedance to the solid mandrel scaffolds has shown that cells seeded on the solid mandrel scaffolding resulted in the typical dense cellular layer restricted to the surface. The seeded air-impedance scaffolds consistently had cells infiltrating approximately half the scaffold thickness (with less than 6 hours interaction with scaffolds) in regions of the pores; cells on adjacent areas were limited to the surface. In summary, the preliminary data demonstrates the feasibility of airimpedance electrospinning and the potential for development of 3-D tissue engineered constructs.

So, are any of these techniques the answer? While the results of the various methods for enhancing electrospun scaffolding porosity are encouraging, much more process optimization and in vitro and in vivo scaffold testing is necessary for a variety of different tissues and organs until any method can truly be considered successful. In many cases, the main question is how much porosity is enough or even too much? This is yet another question that requires a great deal of evaluation. Regardless, without three-dimensional functional tissue regeneration, electrospun scaffolds will not be considered successful. Overall, one safe bet is that a single processing technique will not be feasible for all tissues and organs, thus one needs to consider the desired characteristics necessary for their tissue engineering approach and select the scaffold fabrication technique to meet the requirements. In conclusion, these porosity enhancing techniques continue to enhance the toolbox available to tissue engineers, and allow the field to exploit further the tremendous potential of the electrospun ECM analogues.

\section{References}

1. Boland ED, Bowlin GL,Wnek GE (2004) Tissue Engineering Scaffolds, in The Encyclopedia of Biomaterials and Biomedical Engineering. Marcel Dekker, Inc. 1630-1638.

2. Ramakrishna S, Fujihara K, Teo WE, Lim TC, Ma Z (2005) Introduction to Electrospinning and Nanofibers. World Scientific Publishing Company, Incorporated.

3. Bowlin GL, Lewandrowski K (2002) Electrospinning of polymer scaffolds for tissue engineering, in Tissue Engineering and Biodegradable Equivalents: Scientific and Clinical Applications. Marcel Dekker, Inc. 165-178.

4. Huang ZM, Zhang YZ,Kotaki M, Ramakrishna S (2003) A review on polymer nanofibers by electrospinning and their applications in nanocomposites. Composites Science and Technology 63: 2223-2253.

5. Sell SA, McClure MJ, Garg K, Wolfe PS, Bowlin GL (2009) Electrospinning of collagen/biopolymers for regenerative medicine and cardiovascular tissue engineering. Adv Drug Deliv Rev 61: 1007-1019.

6. Madurantakam PA, Cost CP, Simpson DG, Bowlin GL (2009) Science of nanofibrous scaffold fabrication: strategies for next generation tissueengineering scaffolds. Nanomedicine (Lond) 4: 193-206.

7. Li M, Mondrinos MJ, Gandhi MR, Ko FK, Weiss AS, et al. (2005) Electrospun protein fibers as matrices for tissue engineering. Biomaterials 26: 5999-6008.

8. Barnes CP (2007) Preliminary investigation of electrospun collagen and polydioxanone for vascular tissue engineering applications. International Journal of Electrospun Nanofibers and Applications 1: 73-87.

9. McManusa MC, Bolandb ED, Kooa HP, Barnesb CP, Pawlowski KJ, et al. (2006) Mechanical properties of electrospun fibrinogen structures. Acta Biomater 2 : 19-28

10. McClure MJ, Sell SA, Simpson DG, Bowlin GL (2009) Electrospun polydioxanone, elastin, and collagen vascular scaffolds: Uniaxial cyclic distension. Journal of Engineered Fibers and Fabrics 4:18-25

11. Zhang Y, Ouyang H, Lim CT, Ramakrishna S, Huang ZM (2005) Electrospinning of gelatin fibers and gelatin/PCL composite fibrous scaffolds. J Biomed Mater Res B Appl Biomater 72: 156-165.

12. Baker BM, Gee AO, Metter RB, Nathan AS, Marklein RA, et al. (2008) The potential to improve cell infiltration in composite fiber-aligned electrospun scaffolds by the selective removal of sacrificial fibers. Biomaterials 29: 2348 2358. 
Citation: Bowlin GL (2010) Enhanced Porosity without Compromising Structural Integrity: The Nemesis of Electrospun Scaffolding. J Tissue Sci Eng 2:103e. doi:10.4172/2157-7552.1000103e

Page 3 of 3

13. Nam J, Huang Y, Agarwal S, Lannutti J (2007) Improved cellular infiltration in electrospun fiber via engineered porosity. Tissue Eng 13: 2249-2257.

14. Leong MF, Rasheed MZ, Lim TC, Chian KS (2009) In vitro cell infiltration and in vivo cell infiltration and vascularization in a fibrous, highly porous poly $(D, L-$ lactide) scaffold fabricated by cryogenic electrospinning technique. J Biomed Mater Res A 91: 231-240.
15. Leong MF, Chan WY, Chian KS, Rasheed MZ, Anderson JM (2010) Fabrication and in vitro and in vivo cell infiltration study of a bilayered cryogenic electrospun poly (D,L-lactide) scaffold. J Biomed Mater Res A 94: 1141-1149.

16. Blakeney BA, Tambralli A, Anderson JM, Andukuri A, Lim DJ, et al. (2011) Cell infiltration and growth in a low density, uncompressed three-dimensional electrospun nanofibrous scaffold. Biomaterials 32: 1583-1590. 